ISSN 1991-8631

Original Paper

http://indexmedicus.afro.who.int

\title{
Evaluation des procédés traditionnels de production du ablo, un pain humide cuit à la vapeur, au Bénin
}

\author{
Joseph DOSSOU ${ }^{1 *}$, Germain Elolo OSSEYI ${ }^{2}$, Franck Kodjo K. AHOKPE ${ }^{1}$ et \\ Sylvanus D. P. ODJO ${ }^{1}$ \\ ${ }^{I}$ Département de Nutrition et Sciences Alimentaires, Faculté des Sciences Agronomiques, Université d'Abomey \\ Calavi, BP: 526 Cotonou, Bénin. \\ ${ }^{2}$ Ecole Supérieure des Techniques Biologiques et Alimentaires de l'Université de Lomé, Togo. \\ "Auteur correspondant, E-mail: jokdossou@yahoo.fr, Tel:+229-97086266.
}

\section{RESUME}

"Ablo" est un pain humide en forme de boulettes cuites à la vapeur et vendues dans les rues au Bénin. La présente étude est réalisée sur ce produit et vise comme objectif de caractériser les différentes technologies de production de cet aliment et d'en analyser la qualité physico-chimique, microbiologique et sensorielle. A cet effet, une enquête technologique a été réalisée, suivie d'une production expérimentale d'échantillons du ablo, soumis aux analyses physico-chimiques, microbiologiques et sensorielles. Il ressort de l'enquête qu'il existe trois procédés de production du ablo, variant suivant les principales matières premières utilisées, à savoir, le maïs, le riz ou un mélange de $2 / 3$ de maïs pour $1 / 3$ de riz. Ablo à base de maïs est reconnu comme le produit original et son procédé de production subsiste et prédomine encore dans les petites agglomérations, tandis que celui à base du riz relève d'une récente modification de la technologie originelle. L'analyse des caractéristiques physico-chimiques révèle que le $\mathrm{pH}$ du ablo, d'une valeur moyenne de 5,01 et son acidité titrable de 2,83\% d'acide lactique dépendent de la durée de fermentation, mais prouvent aussi que ce produit est un aliment légèrement acide. Les teneurs en matière sèche et en protéines brutes sont respectivement de $39 \%$ et 5,2\%, tandis que celles des cendres brutes et des sucres totaux sont estimées à $1,1 \%$ et à $3,1 \%$. L'étude a également révélé que la fermentation est assurée par les levures et les bactéries lactiques. Par rapport à sa qualité sanitaire, ablo peut être considéré comme un aliment hygiénique à cause de l'absence de germes pathogènes due à son acidité et à sa cuisson à la vapeur à une température de $95{ }^{\circ} \mathrm{C}$ environ. Toutefois, il peut être contaminé par la microflore ambiante au cours des manipulations post-cuisson.

(C) 2011 International Formulae Group. All rights reserved.

Mots clés : Bénin, ablo, évaluation, procédés artisanaux, qualité.

\section{INTRODUCTION}

Les régimes alimentaires des peuples africains sont essentiellement basés sur des ressources d'origine végétale dont en particulier les céréales. En effet, dans la région ouest africaine, les céréales les plus consommées sont, par ordre d'importance, le maïs, le riz, le mil et le sorgho; au Bénin, le mais occupe une place de choix dans l'alimentation des populations; ceci explique le niveau significatif de consommation estimée à $85 \mathrm{~kg} / \mathrm{habitant} / \mathrm{an}$ (Nago,1997), avec 
des valeurs allant de moins de 30 $\mathrm{kg} / \mathrm{habitant} / \mathrm{an}$ dans le septentrion à 100 et 150 $\mathrm{kg} / \mathrm{habitant} / \mathrm{an}$ dans le sud et le centre du Bénin (Nago, 1989 ; Hounhouigan, 1994 ). La consommation du riz est aussi en plein essor au Bénin. Estimée à une moyenne de 14 kg/habitant/an en 1999 (INSAE, 2000), elle a connu un taux de croissance de $46 \%$ entre 2000 et 2005 et atteint de nos jours plus de 40 $\mathrm{kg} /$ habitant/an (Djakpo, 2010). Le maïs et le riz subissent le plus souvent des transformations artisanales ou semiindustrielles avant d'être consommés; les principaux produits obtenus de premières transformations du maïs sont la farine sèche linfin, puis des pâtes fermentées comme ogui et mawê (Hounhouigan, 1994). D'une manière générale, la préparation de ces produits issus des céréales reste encore artisanale et la technique de préparation se transmet de mère en fille. A partir du mawê, sont obtenus divers produits dont les pâtes cuites fermentées, les semoules, les bouillies fermentées et les beignets. L'un des produits obtenus à partir du mawê du maïs et/ou du riz est ablo, qui est une pâte fermentée et cuite. Ablo est un aliment de rue car il est préparé et vendu spécialement dans les lieux publics, sous forme d'aliment prêt à la consommation. Ainsi, comme tout aliment de rue, la production du ablo est sujette à de nombreuses contraintes dont notamment ce qui suit (Aholou-Yèyi, 2007 ; Odjo, 2008) : une forte variabilité au niveau de la qualité marchande du produit fini, due à la diversité des procédés de production et la non maîtrise des paramètres technologiques; une courte durée de conservation du produit fini dont les caractéristiques physico-chimiques et organoleptiques changent très vite dans les conditions actuelles de conservation et de commercialisation; l'utilisation d'un équipement rudimentaire à faible rendement de production, parfois constitué de matériau de récupération et qui peut contribuer à la contamination chimique du produit au cours de la préparation; l'utilisation d'emballages inappropriés comme des sachets plastiques et des boîtes de concentré de tomate récupérées et découpées et surtout le manque de connaissances scientifiques sur les procédés de production et la qualité du ablo.

En effet, malgré l'importance socioéconomique du ablo au sud du Bénin et l'essor remarquable que connaît sa consommation ces dernières années sur toute l'étendue du territoire national et dans certains pays de la sous-région comme le Togo, le Ghana, la Côte d'Ivoire et le Nigeria (Dossou et al., 2008), très peu d'études ont été consacrées à ce produit. Il convient donc d'identifier et d'évaluer les procédés traditionnels de production du ablo. Par conséquent, la présente étude vise une meilleure connaissance des systèmes techniques de production et la valeur nuritive $\mathrm{du}$ ablo. Plus spécifiquement, il s'agit d'identifier les différents procédés traditionnels de fabrication du ablo, de caractériser les paramètres physico-chimiques et microbiologiques des différents types de ablo et d'en évaluer la qualité sensorielle.

\section{MATÉRIEL ET MÉTHODES}

L'étude est réalisée sur la base d'enquête pour l'identification et la caractérisation des principaux procédés de fabrication du ablo. Ensuite, des échantillons du ablo produits et prélévés chez l'une des productrices traditionnelles enquêtées sont analysés au laboratoire.

\section{Enquête}

Sur la base des informations préliminaires recueillies sur la zone d'étude, les villes de Cotonou, de Comè et de Grandpopo sont les trois (3) principales localités au Sud-Bénin retenues pour l'enquête. Dans ces localités, l'activité de production-vente et la consommation du ablo sont prédominantes. L'enquête s'est adressée aux productrices traditionnelles du ablo, à raison de 30 par localité. Des données de l'enquête sont collectées à l'aide d'un guide d'entretien sur les aspects socio-culturels de la production, les séquences et la nature des opérations unitaires, les matières premières et les facteurs déterminant leur choix, les caractéristiques du 
matériel et les paramètres de production, l'appréciation de la qualité sensorielle des produits intermédiaires et du ablo, etc. L'enquête a permis d'élaborer le diagramme technologique de production et de prélever des échantillons du ablo pour les analyses de laboratoire.

\section{Analyse des échantillons du ablo}

Une fois les systèmes techniques de production inventoriés et décrits, une productrice ayant une bonne maîtrise de la technologie a été identifiée pour réaliser, selon la méthode traditionnelle, les échantillons du ablo destinés aux analyses de laboratoire. Ainsi des échantillons de pâte de fermentation et du ablo ont été prélevés en trois répétitions, respectivement pour l'étude de la dynamique de fermentation et pour les analyses de la qualité nutritionnelle et sensorielle d'ablo. Pour la dynamique de fermentation, les échantillons ont été prélévés à des intervalles d'une heure pendant toute la durée de la fermentation. Les échantillons ont été ramenés au laboratoire pour les analyses immédiatement après leur production.

\section{Analyses physicochimiques}

Des analyses physico-chimiques sont effectuées pour connaître la composition biochimique et la valeur nutritionnelle du ablo. La teneur en eau est déterminée suivant la méthode AACC, 44, 15 A (AOAC, 1984), à partir de $5 \mathrm{~g}$ d'échantillon frais, par pesée différentielle, après passage à l'étuve Heraeus $\mathrm{T} 5042$ à $105{ }^{\circ} \mathrm{C}$ pendant $72 \mathrm{~h}$. Le pH et l'acidité titrable sont déterminés suivant la méthode de Nout modifiée (AOAC, 1984). La teneur en protéines est déterminée selon la méthode de Kjeldahl après la minéralisation d'un gramme d'échantillon dans un digesteur sous une hotte à $420{ }^{\circ} \mathrm{C}$, puis la distillation dans un distillateur d'azote et la titration avec l'acide chlorhydrique à $0,1 \mathrm{~N}$. La teneur en cendres est évaluée suivant la méthode AACC, 08-01 (AOAC, 1984), par la carbonisation puis l'incinération au four à moufle à $550{ }^{\circ} \mathrm{C}$ pendant $24 \mathrm{~h}$. Les sucres totaux sont déterminés suivant la méthode
Luff-Schoorl (Lees, 1968), après l'extraction des sucres avec de l'éthanol à $40 \%$ (v/v), la défécation au moyen des réactifs Carrez 1 et Carrez 2, l'inversion du saccharose puis la titration des sucres totaux avec le thiosulfate de sodium à $0,1 \mathrm{~N}$.

\section{Analyses microbiologiques du ablo}

Les analyses microbiologiques ont consisté au dénombrement de la flore aérobie mésophile (sur PCA CM 0325 Oxoid et incubée pendant 72 heures à $30{ }^{\circ} \mathrm{C}$ ), des bactéries lactiques (sur MRS-agar en double couche à $30^{\circ} \mathrm{C}$ pendant $72 \mathrm{~h}$ ), des levures (sur OGA pendant 5 jours) et les entérobactéries (sur VRBG en double couche à $37^{\circ} \mathrm{C}$ ). Les prélèvements des échantillons analysés ont été faits toutes les heures pendant les dix heures qu'a duré la fermentation expérimentale. Les bactéries lactiques et les levures ont été choisies car ce sont elles qui assurent principalement la fermentation des céréales, tandis que les entérobactéries rendent compte de la qualité hygiénique du produit.

\section{Analyse sensorielle}

Un test de préférence sur les trois types de "ablo" identifiés au cours de l'enqête a été réalisé sur un panel de 60 dégustateurs pour déterminer le produit le plus apprécié par rapport aux critères goût, couleur, odeur et texture. Des scores variant de 1 à 5 ont été attribués à ces critères; ablo préféré étant celui ayant le meilleur score.

Pour l'ensemble des analyses, les données recueillies ont été traitées au logiciel Microsoft Excel 2003. Une analyse de variance a été réalisée pour apprécier le degré de significativité des valeurs obtenues.

\section{RESULTATS}

Caractéristiques socio-culturelles de la production du ablo

L'enquête réalisée dans les villes de Comè, Grand Popo et Cotonou, a révélé que ablo est un aliment consommé traditionnellement dans l'aire géographique des peuples Mina et Houeda, établis depuis plusieurs siècles dans la zone littorale du sud- 
ouest du Bénin. Il relève donc du patrimoine culturel et culinaire de ces peuples. Ablo est un aliment préparé à base de mawè, une pâte fermentée de maïs, panifiée, cuite à la vapeur et présentée sous forme de boulettes ovoïdes, blanchâtres et de structure alvéolaire. Ablo résulte d'un procédé de fermentation panaire, suivi d'une cuisson à la vapeur humide et peut donc être considéré comme un "pain humide". Il est connu sous des appellations locales diverses, dont blou et ablou en Adjagbé, une langue parlée au Sud-Ouest du Bénin par le groupe socio-culturel Adja. Or, selon la tradition orale adja, ablou dérive du ablou-kpoma, qui est une pâte fermentée cuite au four à pain. Ceci témoigne de l'évolution que la technologie aurait subie dans le temps et confirme l'appellation de "pain humide" qui lui est attribuée ici. De nos jours, ablo est principalement produit et consommé dans les villes de Grand Popo, Agoué et Comè, d'où il s'est disséminé dans les grandes villes du Bénin, en particulier, Ouida, Cotonou et Porto-Novo. Vendu souvent sur les voies et/ou places publiques, ablo appartient donc au groupe des aliments de rues, dont l'enjeu pour la santé des consommateurs est préoccupant (FAO/OMS, 2005). D'un point de vue socioculturel, ablo constitue un aliment très apprécié, souvent consommé avec du poisson ou de la viande frite d'aulacode, associée à une sauce de piment et de tomate. Il est utilisé lors des cérémonies rituelles et funéraires de certaines familles. Il est aussi consommé lors des grandes réjouissances (baptême, mariage, anniversaire, fêtes populaires, etc.). Aujourd'hui, ablo revêt un caractère commercial prééminent et donne lieu à une production manufacturière en plein essor. Néanmoins, cette production marchande du ablo demeure encore une activité exclusivement féminine, même si certaines étapes de la chaîne de production (spécifiquement la mouture de la céréale) sont réalisées sur prestation par des hommes. Pour l'ensemble des personnes enquêtées, l'activité se réalise dans de petites unités de type familial où ce savoir-faire se transmet donc de mère en fille; le niveau de production dans ces unités reste encore artisanal avec une capacité variant entre 10 et $20 \mathrm{~kg}$ du ablo par jour. Cette activité n'entretient qu'une économie domestique de subsistance aujourd'hui. Cependant, les longues files d'attente des clients sur les lieux de production du ablo, surtout dans les grandes villes, témoignent de l'affection et de l'engouement des consommateurs pour cet aliment; ainsi, au cours des deux dernières décennies, il s'est diffusé sur toute l'étendue du territoire béninois, et voire dans certains pays de l'hinterland où il s'impose comme un aliment exotique. De ce point de vue, ablo constitue donc un produit à très fort potentiel économique et dont la valorisation peut générer d'importants revenus et de nombreux emplois féminins durables pour les productrices.

\section{Systèmes techniques de production du ablo}

A l'issue de l'enquête, 3 procédés de fabrication du ablo ont été identifiés se différenciant principalement de par la matière première utilisée : un procédé originel à base de maïs, un procédé modifié à base de riz et un procédé intermédiaire à base d'un mélange de $2 / 3$ de maïs pour $1 / 3$ de riz ont été distingués. Originellement, le maïs était la seule céréale utilisée pour la production du ablo. Ce procédé originel est encore préservé à Comè. C'est d'ailleurs la seule des localités enquêtées où ce type de procédé est rencontré. Bien que la plupart des transformatrices aient reconnu une certaine facilité dans l'utilisation du procédé modifié, le riz n'est pas utilisé ici pour la production du ablo. Les transformatrices évoquent la cherté de cette matière première par rapport au maïs (Odjo, 2008). Pourtant le procédé à base de maïs seul comporte une fastidieuse étape de préparation de la pâte à fermenter, le mawè. Le processus de fabrication du mawè (Figure 1) implique le concassage du maïs préalablement trié et nettoyé, l'élimination du son par trempage et flottaison et la mouture subséquente du gritz obtenu. Le tiers du mawè 
ainsi obtenu est prélevé, malaxé dans l'eau, précuit à une température de 90 à $95{ }^{\circ} \mathrm{C}$ et refroidi avant d'être mélangé au $2 / 3$ restant. Ainsi, la pâte qui en résulte est fermentée et cuite à la vapeur d'eau (Figure 2). La pénibilité et la trop longue durée d'au moins $24 \mathrm{~h}$ des opérations unitaires de ce procédé ont contraint certaines productrices des grandes villes comme Cotonou et Porto-Novo à substituer progressivement le riz au maïs. Il en résulte un procédé plus simple (Figure 3) puisque l'étape de préparation du mawè est supprimée, réduisant ainsi la durée de production de 6 à 8 h. Le procédé intermédiaire utilise comme matières premières de base $1 / 3$ de riz mélangé à $2 / 3$ de maïs pour la production du mawè. Ces proportions ne sont pas constantes, mais la quantité de riz n'excède guère $40 \%$. Le maïs et le riz sont traités séparément, le premier pour obtenir le mawè, le second pour obtenir la farine fine. Ces deux produits intermédiaires sont ensuite mélangés. Ahokpè (2005) indique que la quantité de mawè représente au plus la moitié du poids du mélange. La pâte qui en résulte est divisée en deux proportions de $1 / 3$ pour $2 / 3$ et le reste des opérations se poursuit jusqu'à l'obtention du ablo. Ce procédé est né du souci des productrices d'atténuer la pénibilité du travail et la durée de production en réduisant la quantité de maïs à traiter tout en visant du ablo de mêmes caractéristiques organoleptiques que celui originel. Dans chacun de ces trois procédés, des additifs et ingrédients sont ajoutés à l'étape de la fermentation panaire (Figures 2 et 3 ). Il s'agit de la farine de blé surtout utilisée pour son réseau de gluten permettant de piéger le $\mathrm{CO}_{2}$ dégagé lors de la fermentation et de développer la structure alvéolaire du ablo, puis le sel et le sucre pour conférer une certaine saveur au produit et la levure de boulangerie (Saccharomyces cerevisiae) ajoutée à la pâte à fermenter pour en accélérer la levée. En résumé, les principales étapes de la fabrication du ablo sont : la préparation de la pâte (mawè), la fermentation panaire et la cuisson à la vapeur. Le matériel de cuisson à la vapeur de la pâte fermentée est constitué de deux grandes bassines en émail retournées l'une sur l'autre et dont les bords sont tapissés d'une couche de morceau de carton ou de linge qui joue le rôle de joint d'étanchéité pour la vapeur de cuisson. A l'intérieur, les portions de pâte fermentée emballées dans des sachets plastiques transparents, sont disposées dans de petits moules métalliques, étalés sur un van. En dessous, la vapeur est produite à partir de l'eau chauffée grâce à un foyer traditionnel alimenté par un feu de bois. Le dispositif fonctionne comme un cuiseur à vapeur. Cet instrument de cuisson est peu efficace car malgré le joint, des bouffées de vapeur s'en échappent toujours. Ceci traduit une perte de chaleur plus ou moins importante au cours de la cuisson, qui réduit ainsi le rendement énergétique du processus. D'autre part, la cuisson est lente, occasionnant souvent une longue file d'attente des clients sur les lieux de production du ablo, surtout dans les grandes villes.

\section{Paramètres technologiques de production du $a b l o$}

La durée de production du ablo varie d'un procédé à l'autre mais aussi en fonction de l'expérience de la productrice puisque la technologie n'est pas standardisée. Elle est estimée en moyenne à 6 heures lorsque le mawè est déjà disponible. La fermentation s'effectue à température ambiante, variant de 28 à $32{ }^{\circ} \mathrm{C}$. Par expérience, les productrices arrêtent la fermentation après 3 à $4 \mathrm{~h}$, puisqu'à cette étape, on observe déjà une production suffisante du dioxyde de carbone $\left(\mathrm{CO}_{2}\right)$ pour la formation d'alvéoles dans le produit fini. Une durée de 3 à $4 \mathrm{~h}$ de fermentation suffit pour obtenir une bonne structure alvéolaire désirée par le consommateur du ablo. La consommation énergétique de la production a été estimée sur la base du bois de teck (Tectona grandis) qui est le combustible le plus souvent utilisé pour la cuisson du ablo. La consommation spécifique en combustible est en moyenne de $2 \mathrm{~kg} / \mathrm{kg}$ de matière 
première, qui équivalent à $2.392,34 \mathrm{~kJ} / \mathrm{kg}$, sur la base d'un pouvoir calorifique de 1196,17 $\mathrm{kJ} / \mathrm{kg}$, comme l'indiquent Dossou et al. (2005) pour le bois de teck. En raison des difficultés d'approvisionnement en bois dans la zone d'étude, cette valeur est déjà inquiétante même si elle est inférieure à celle de la cuisson du gari jugée excessive, soit 2,69 $\mathrm{kg} / \mathrm{kg}$ de matière première (Dossou et al., 2005) et comparable à celle de la cuisson de chakpalo qui est de $2,4 \mathrm{~kg} / \mathrm{kg}$ de chakpalo (Dossou et Ballogou, 2007).

Le rendement en ablo varie en fonction des procédés et dépend surtout des traitements subis par les matières premières. Le rendement en ablo est de $80,3 \%$ pour le procédé à base de maïs où le plus fort taux de pertes de matières dues aux opérations de concassage et surtout de séparation du son est enregistré. Par contre, le rendement est de $85,7 \%$ pour ablo à base de riz et de $83,1 \%$ pour ablo à base du mélange de maïs et du riz.

\section{Fermentation panaire de la pâte du ablo}

La fermentation de type panaire subie par la pâte du ablo est déclenchée par l'ajout de la levure boulangère et vise la levée rapide de la pâte suivie d'une acidification. La dynamique microbienne au cours de cette fermentation est étudiée pour ablo au riz. La flore aérobie mésophile, les levures et les bactéries lactiques sont déterminées pendant $10 \mathrm{~h}$ de fermentation. La flore aérobie mésophile, les levures et les bactéries lactiques augmentent de façon graduelle jusqu'à la troisième heure (Figure 4). Cette phase peut être assimilée à la phase logarithmique de croissance des microorganismes, qui correspond à l'étape de production maximale de dioxyde de carbone $\left(\mathrm{CO}_{2}\right)$, provenant de l'oxydation des sucres fermentescibles; ensuite intervient un léger ralentissement de la croissance des microorganismes de la cinquième à la sixième heure. La population microbienne tend alors à se stabiliser jusqu'à la huitième heure. Par contre les entérobactéries sont absentes du produit.

\section{Qualité nutritionnelle du ablo}

La teneur des principaux groupes de nutriments est déterminée dans les différents types du ablo afin d'apprécier leur valeur nutritionnelle. Ablo est un aliment légèrement acide, mais dont le $\mathrm{pH}$ et l'acidité varient en fonction de la matière première utilisée (Tableau 1). Ablo à base de riz est le moins acide avec la plus forte valeur de $\mathrm{pH}$ et la plus faible acidité titrable. Ablo à base de riz a les plus fortes teneurs en lipides, en sucres réducteurs et en cendres, mais enregistre aussi le plus faible taux de protéines (Tableau 1). Ablo à base du mélange maïs et riz a des valeurs intermédiaires entre ablo à base de maïs et ablo à base de riz.

\section{Qualité sensorielle et préférence du consommateur du ablo}

Un test de préférence réalisé sur un panel de 60 dégustateurs a montré que ablo à base de riz est le plus apprécié par les consommateurs (Tableau 2). Cette préférence des consommateurs pour ablo à base de riz justifie l'engouement que suscite actuellement la production de ce type du ablo, en particulier dans les grandes villes. Cependant, cet engouement ne se traduit pas par une augmentation de la consommation du riz local. Au contraire, la présence du riz local dans les grands centres de consommation est marginale et ne représente que 10 à $15 \%$ des importations de riz (Abiassi et Echou, 2006). Les raisons évoquées par les transformatrices pour justifier la non utilisation des variétés de riz produit localement sont indiquées dans le Tableau 3. La principale cause évoquée par la majorité des productrices enquêtées a rapport à la couleur terne des variétés de riz local. 
Tableau 1 : Caractéristiques physicochimiques des différents types du ablo.

\begin{tabular}{|c|c|c|c|c|c|c|c|c|}
\hline $\begin{array}{c}\text { Type } \\
\text { de } a b l o\end{array}$ & Matière sèche (\%) & pH & $\begin{array}{l}\text { Acidité } \\
\text { titrable }^{1}\end{array}$ & Protéines $^{2}$ & Lipides $^{2}$ & $\begin{array}{c}\text { Sucres } \\
\text { totaux }\end{array}$ & $\begin{array}{c}\text { Sucres } \\
\text { réducteurs }\end{array}$ & Cendres $^{2}$ \\
\hline ablo riz & $\begin{array}{c}32,77 \pm \\
3,94 \mathbf{a}\end{array}$ & $5,28 \pm 0,015 \mathbf{a}$ & $1,25 \pm 0,07 \mathbf{a}$ & $7,44 \pm 0,14 \mathbf{a}$ & $1,50 \pm 0,055 \mathbf{a}$ & $10,46 \pm 0,66 \mathbf{a}$ & $2,71 \pm 0,21 \mathbf{a}$ & $2,15 \pm 0,18 \mathbf{a}$ \\
\hline ablo maïs-riz & $39,90 \pm 0,39 \mathbf{a}$ & $4,85 \pm 0,015 \mathbf{c}$ & $1,64 \pm 0,08 \mathbf{a b}$ & $7,82 \pm 0,18 \mathbf{a}$ & $\begin{array}{c}1,07 \pm \\
0,060 \mathbf{a b}\end{array}$ & $11,15 \pm 0,45 \mathbf{a}$ & $1,09 \pm 0,09 \mathbf{b}$ & $2,09 \pm 0,06 \mathbf{a}$ \\
\hline ablo maïs & $35,62 \pm 3,61 \mathbf{a}$ & $4,66 \pm 0,020 \mathbf{b}$ & $2.09 \pm 0,09 \mathbf{b}$ & $11,01 \pm 0,09 \mathbf{b}$ & $0.79 \pm 0,125 \mathbf{b}$ & $15,31 \pm 0,24 \mathbf{b}$ & $0,91 \pm 0,18 \mathbf{b}$ & $2,33 \pm 0,32 \mathbf{a}$ \\
\hline
\end{tabular}

N.B : ${ }^{1}$ En pourcentage d'acide lactique ; ${ }^{2}$ Valeur rapportée à la matière sèche. Les valeurs portant des lettres différentes dans la même colonne sont significativement différentes au seuil de $5 \%$.

Tableau 2 : Note sensorielle attribuée aux différents types du ablo.

\begin{tabular}{lc}
\hline \multicolumn{1}{c}{ Type de "ablo" } & Score attribué \\
\hline Ablo à base de riz & $4,59 \pm 0,83 \mathbf{a}$ \\
Ablo à base de maïs & $2,04 \pm 0,82 \mathbf{b}$ \\
Ablo à base de maïs et de riz & $4,09 \pm 1.08 \mathbf{c}$ \\
\hline N.B : $1: 2 / 3$ de maïs et $1 / 3$ de riz. Les valeurs portant des lettres différentes \\
dans la même colonne sont significativement différentes au seuil de $5 \%$.
\end{tabular}

Tableau 3 : Raisons évoquées pour la non utilisation du riz pour la production du ablo

\begin{tabular}{lc}
\hline Motifs & Pourcentage de productrices \\
\hline Non disponibilité du riz local & $43,75 \%$ \\
Couleur sale du riz local & $93,75 \%$ \\
Faible capacité d'absorption d'eau & $61 \%$ \\
Taux de brisures élevé & $6,25 \%$ \\
\hline
\end{tabular}




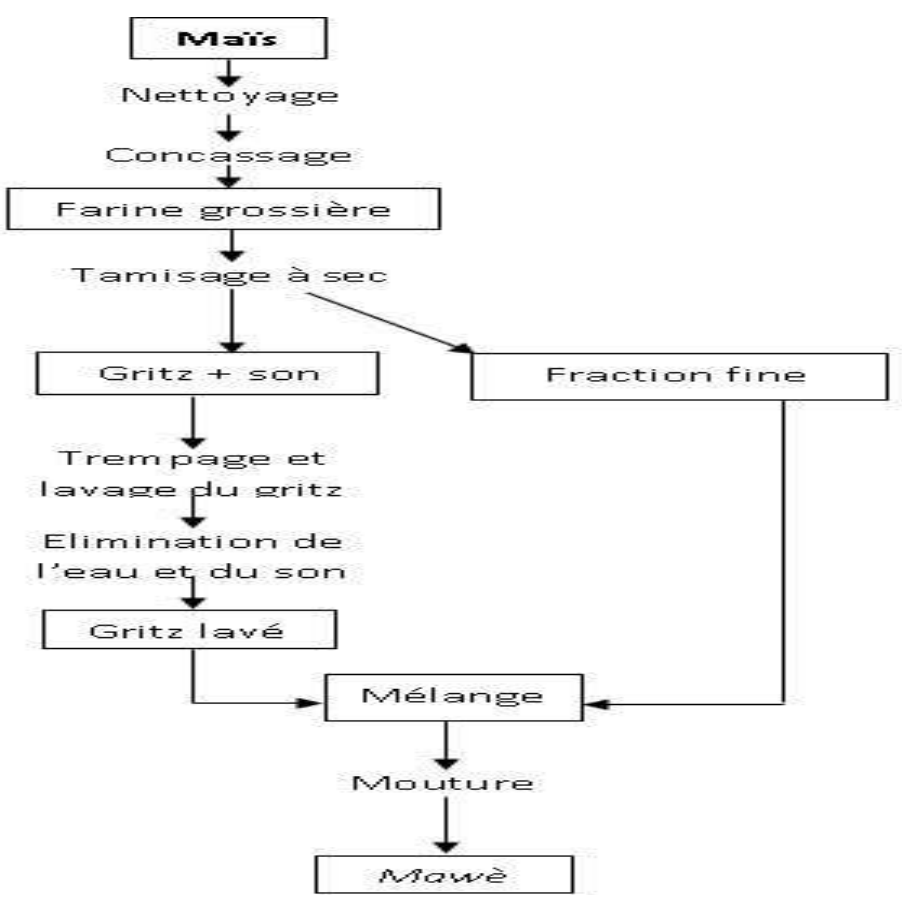

Figure 1 : Diagramme de fabrication du mawè à partir du maïs

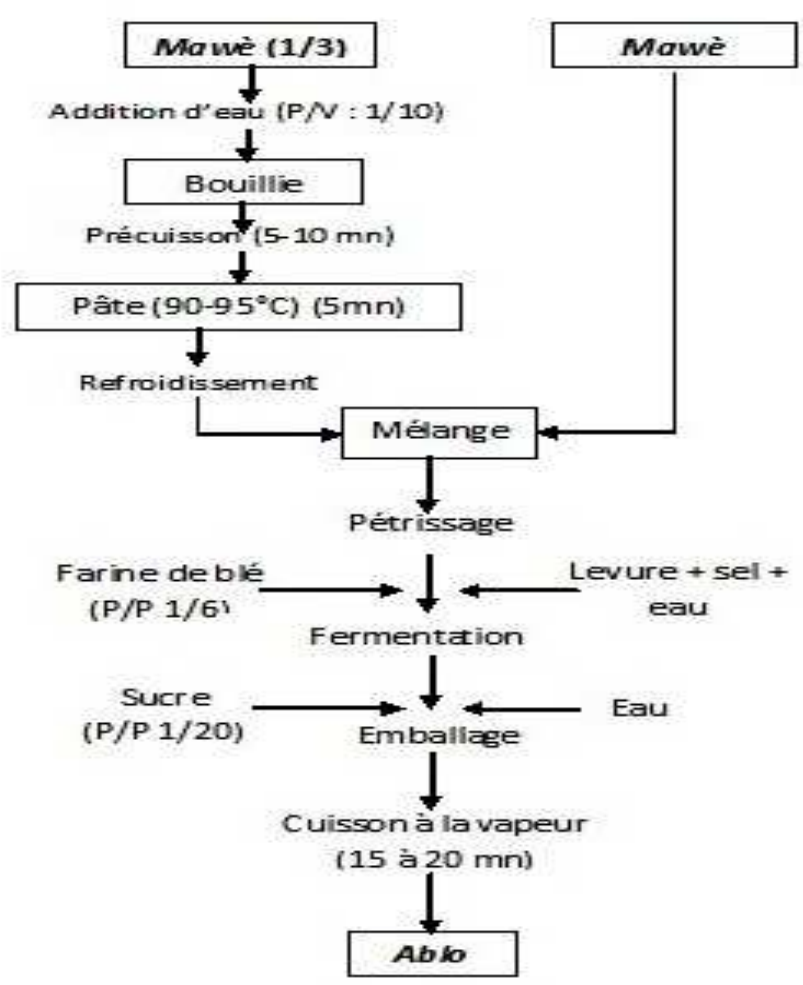

Figure 2 : Diagramme de Fabrication du ablo à partir du mawè. 


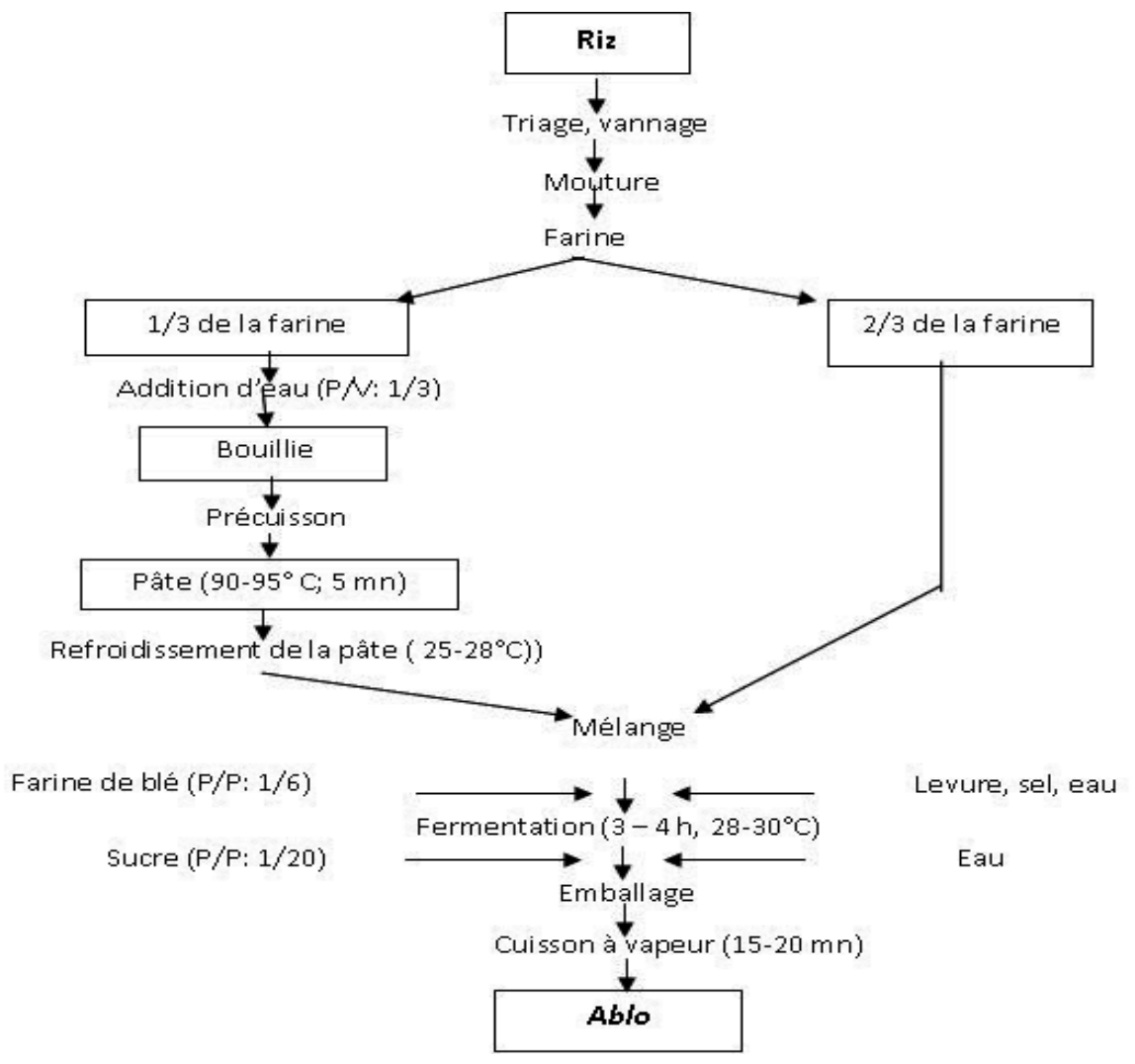

Figure 3 : Diagramme de fabrication du ablo à partir du riz.

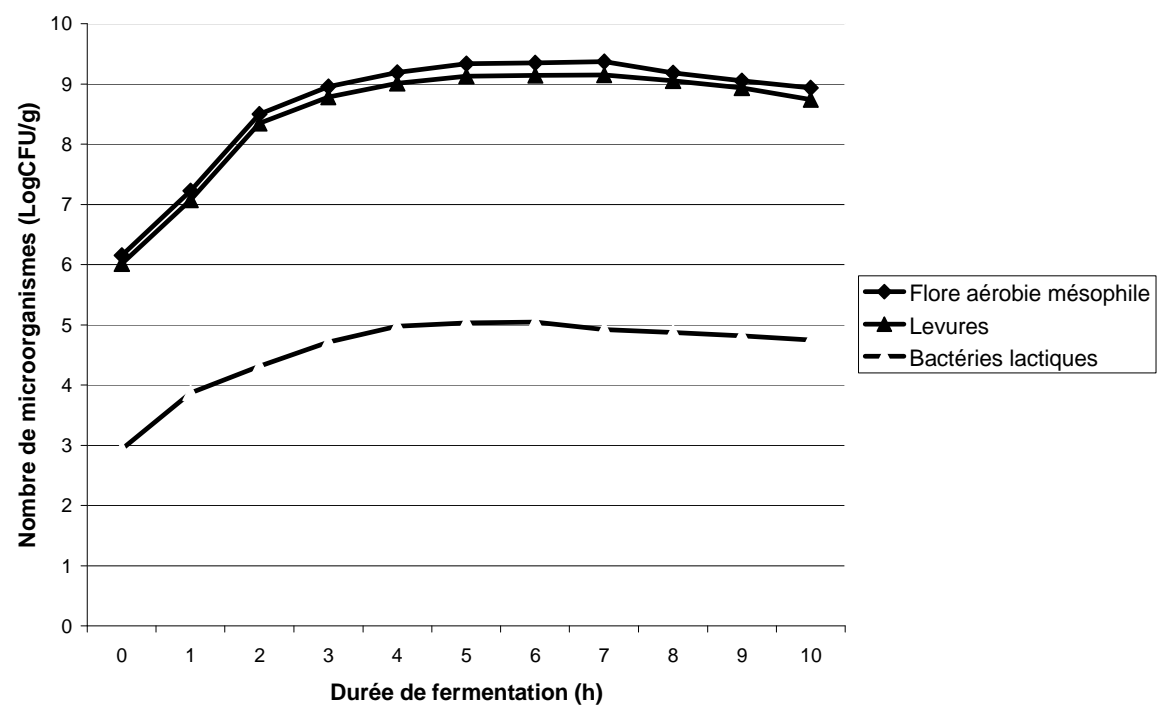

Figure 4: Évolution de la flore aérobie mésophile, des levures et bactéries lactiques au cours de la fermentation du ablo. 


\section{DISCUSSION}

L'étude visait à identifier les différents procédés traditionnels de fabrication du ablo, à caractériser les paramètres physicochimiques et microbiologiques des différents types du ablo et à évaluer la qualité sensorielle. A l'issue de l'enquête, trois procédés de fabrication du ablo ont été identifiés se différenciant principalement de par la matière première utilisée: un procédé originel à base de maiis, un procédé modifié à base de riz et un procédé intermédiaire à base d'un mélange de $2 / 3$ de maïs pour $1 / 3$ de riz ont été distingués. Les travaux de Ahokpè (2005) et de Aholou-Yèyi (2007) confirment aussi l'existence de ces trois procédés. Le procédé identifié ici pour la production du ablo à base de maïs est similaire à celui décrit par Hounhouigan (1997). Toutefois, cet auteur ne fait pas cas des possibilités de production du ablo à partir du riz ou d'un mélange maïs et riz. Ce qui montre que cette pratique est assez récente et s'est certainement développée principalement dans les grandes agglomérations urbaines pour répondre à une demande sans cesse croissante en restauration rapide pour des citadins. Le rendement en $a b l o$ varie en fonction des procédés et dépend surtout des traitements subis par les matières premières. Le procédé à base de maïs est caractérisé par le plus fort taux de pertes de matières dues aux opérations de concassage et surtout de séparation du son donnant ainsi les plus faibles rendements. L'étude de la dynamique microbienne au sein des différents types de ablo a montré que les populations des bactéries lactiques et des levures évoluent en parallèle. Les levures prédominent dans la pâte et sont les principaux responsables de la fermentation du ablo. Le développement des bactéries lactiques est probablement favorisé par l'activité des levures qui leur fournissent des composés azotés et des facteurs de croissance comme la vitamine B (Nout et al., 1989). En retour, l'acidification du milieu créée par les bactéries lactiques est favorable au développement des levures. Cette association symbiotique entre les levures et les bactéries lactiques est notée dans la fermentation du mawè (Hounhouigan, 1994), dans la fermentation du gowé (Mitchodjehoun-Mestres et al., 2005) et dans la fermentation du chakpalo (Ballogou, 2008). Par contre, l'acidification du milieu est défavorable au développement des entérobactéries dont le nombre décroît dans la pâte en fermentation (Ahokpè, 2005). Aucun germe pathogène n'est déterminé dans ablo. Ce produit peut être considéré comme un aliment hygiénique à cause de l'absence de germes pathogènes due à son acidité et à sa cuisson à la vapeur à une température de 95 ${ }^{\circ} \mathrm{C}$ environ. Toutefois, les études de Ahokpè (2005) et Aholou- Yèyi (2007) prouvent qu'il peut être contaminé par la microflore ambiante au cours des manipulations postcuisson. Par ailleurs, l'utilisation de sachets en plastique non alimentaire pour emballer le ablo demeure une source inquiétante de contamination chimique non encore élucidée. Selon Djakpo (2010), il s'agit là d'une pratique récente observée chez certaines productrices surtout dans les grandes villes, alors que ablo originel est emballé dans des feuilles de Thalia welwichii.

L'appréciation de la qualité nutritionnelle des différents types de ablo a montré que Ablo à base de riz a les plus fortes teneurs en lipides, en sucres réducteurs et en cendres, mais aussi, le plus faible taux de protéines. Les faibles teneurs en protéines enregistrées au niveau du ablo à base de riz et du mélange maïs et riz peuvent être expliquées par l'utilisation du riz importé qui a été usiné avant sa commercialisation. L'utilisation des variétés locales de riz pour la production du ablo constitue donc un atout sur le plan nutritionnel, puisqu'elles peuvent permettre de relever la teneur en protéines de cet aliment. Ablo à base de riz est le moins acide avec la plus forte valeur de $\mathrm{pH}$ et la plus faible acidité titrable. La baisse $\mathrm{du} \mathrm{pH}$, qui 
correspond à l'augmentation de l'acidité titrable résulte du métabolisme des sucres fermentescibles par les bactéries pour produire de nombreux métabolites dont l'acide lactique. Ces résultats sont conformes aux hypothèses de Niché et al. (1994) formulées sur la fermentation du "kenkey", une pâte fermentée d'origine ghanéenne à base de maïs, aux résultats trouvés par Mugula et al. (2002) sur le "togya", un produit tanzanien à base de sorgho malté et non malté ainsi qu'aux résultats de Mitchodjèhoun et al. (2005) sur le "gowé". Toutes ces observations enregistrées au cours de la fermentation sont caractéristiques des farines de céréales en fermentation (Hounhouigan, 1994 ; Odounfa et Adelèyé, 1985). Ceci s'explique par le fait que la durée de fermentation est plus courte dans le cas du ablo à base de riz que dans les autres cas (Ahokpè, 2005; Aholou-Yèyi, 2007).

L'analyse sensorielle effectuée a montré que ablo à base de riz est le plus apprécié par les consommateurs. Cependant ce type du ablo n'est pas produit à base du riz local. Les productrices du ablo choisissent du riz importé et se justifient sur la base des critères suivants : (1) le riz doit être bien sec et avoir un bon rendement en farine (riz farineux) ; (2) le riz doit être riche en amidon ; (3) le riz doit avoir une forte capacité d'absorption d'eau. Le choix dépend aussi du prix du riz sur le marché local. La principale cause évoquée par la majorité des productrices enquêtées a rapport à la couleur terne des variétés de riz local. En effet, à cause de leur étuvage et surtout parce que les grains n'ont pas subi un polissage, ces variétés de riz paraissent moins blanches que celles importées. Les transformatrices craignent alors d'en obtenir du ablo de couleur moins attrayante, impliquant ainsi une réticence du consommateur et une mévente du produit. Cette perception sensorielle n'est fondée que sur l'arbitraire car au contraire l'étuvage renforce la qualité nutritionnelle du riz en diffusant dans le grain des minéraux et des vitamines (Angladette, 1966; Gariboldi, 1986 ; Houssou, 2002, 2005; Nout et al., 2003 ; ADRAO, 2005, 2008; Dagan, 2008) et les pertes de nutriments occasionnées par le polissage des grains sont évitées au niveau des variétés locales de riz étuvé. La faible capacité d'absorption d'eau des riz locaux est aussi mise en cause, à juste titre, par les productrices du ablo car en réalité les variétés locales de riz sont réputées absorber très peu d'eau. Parmi les 5 variétés locales de riz étudiées (Nerica 1, Nerica 6, TOX 3081, BL 19 et Béris 21), Odjo (2008) précise que seule la variété TOX 3081 a l'indice de réhydratation le plus élevé, soit $76,5 \mathrm{ml}$, qui du reste, est très faible par rapport à la valeur de 96,5 ml trouvée pour la variété de riz importé "spécial rice", utilisée pour la production du ablo. La plupart des raisons évoquées par les productrices contre les variétés locales sont basées sur des préjugés, puisque les travaux de Odjo (2008) et Djakpo (2010) démontrent que BL 19 et TOX 3081, qui sont notamment des variétés locales de riz, ont de très bonnes aptitudes à la production du ablo. D'ailleurs, ablo produit à partir de la variété BL 19 est le plus apprécié par les dégustateurs tandis que ablo obtenu à partir de la variété TOX 3081 présente l'avantage d'être plus riche en nutriments. Ces observations ravivent la problématique de la compétitivité du riz local. Si la compétitivité est comprise ici comme étant l'aptitude du produit considéré à satisfaire, mieux que son concurrent, les besoins et les attentes de ses utilisateurs en terme de meilleure qualité, meilleur rapport qualité/prix et meilleur attrait (Abiassi et Echou, 2006), alors, des efforts de promotion restent à déployer pour rendre le riz local plus compétitif. C'est dans ce cadre que s'inscrivent les initiatives de la FAO (FAO, 1997) et d'autres acteurs (Hounhouigan, 2006; MAEP, 2005 ; Gbenou, 2004; CCRB, 2004). Certes, l'ONASA estime le prix du riz local avec un taux de brisure de l'ordre de 30 à $35 \%$ 
plus bas que celui de son équivalent importé (ONASA, 2005a, 2005b); mais il faut reconnaître que les rapports de forces sur le marché national sont encore en défaveur du riz local. C'est ce que confirme l'analyse de Adégbola et Singbo $(2005,2003)$, qui retiennent qu'au Bénin «la filière riz fonctionne comme si les producteurs locaux sont taxés par les autres acteurs du système », notamment les importateurs de riz. Ce jugement ne dispense pas des efforts à consentir pour l'amélioration de la qualité du riz local, puisque selon Verlinden et Soulé (2003), même si le riz produit localemnt au niveau des principaux périmètres rizicoles (Malanville, Dévé, Kousi-Lélé) comporte moins d'impuretés par rapport à celui des basfonds et décortiqué à l'aide des moulins polyvalents, la qualité de la quasi-totalité du riz local demeure encore inférieure à celle du riz importé du marché international.

\section{Conclusion}

Ablo est un aliment de rue produit à partir du maïs et/ou du riz. La production et la commercialisation de cet aliment constituent une importante activité génératrice de revenus et pratiquée exclusivement par les femmes dans les localités choisies pour la présente étude. Ablo originel est produit à base de maïs, mais la pénibilité et la trop longue durée de ce procédé originel entraînent une modification technologique, se traduisant par une substitution partielle ou totale du maïs par le riz surtout dans les grandes villes. Cependant, bien que le Bénin produise du riz ayant de bonnes aptitudes à la production du ablo, les transformatrices continuent d'utiliser le riz importé dans le procédé modifié. La préférence du riz importé pour la production du ablo peut constituer un frein à la promotion et la consommation des variétés locales de riz. L'amélioration de la performance de l'équipement de cuisson à vapeur, l'étude approfondie de la dynamique de fermentation, la stabilisation et l'élaboration d'une norme de qualité du ablo restent encore des aspects scientifiques et techniques de la production du ablo à étudier.

\section{REFERENCES}

Abiassi EH, Eclou SD. 2006. Etude sur les instruments de régulation des importations commerciales de riz au Bénin. Rapport d'étude, MAEP, Cotonou, 85 p.

Adegbola P, Singbo A. 2005. Impact de l'importation du riz sur la compétitivité et la rentabilité de la production nationale au Bénin. Communication à l'atelier régional de l'ADRAO sur le thème : Politique et stratégies pour la promotion de la production rizicole et la sécurité alimentaire en Afrique subsaharienne. PAPA-INRAB, Bénin.

Adegbola P, Singbo A. 2003. Compétitivité de la filière riz du Bénin dans l'économie internationale ; Rapport d'étude ; MAEP, INRAB, Cotonou, $21 \mathrm{p}$.

ADRAO. 2008. Initiative Africaine sur le Riz (ARI), Rapport d'activités 2003-2007 ADRAO ; Cotonou, Bénin.

ADRAO. 2005. Test de performance de nouveaux dispositifs d'étuvage de riz dans les zones de Glazoué, de Dassa et de Ouèssè. http://www.warda.org/ workshop/aw2005/contents.htn 20.07.2008.

Ahokpe KF. 2005. Valorisation des aliments traditionnels locaux : Evaluation des procédés traditionnels de préparation de "ablo", une pâte fermentée cuite à la vapeur. Mémoire pour l'obtention de la maîtrise professionnelle en Biotechnologies agroalimentaires. Laboratoire de Microbiologie et de Technologies Alimentaires. Faculté des sciences et techniques, Abomey-Calavi, Bénin, 51p.

Aholou-Yèyi AAM. 2007. Evaluation du système technique artisanal de production d'ablo, un pain béninois cuit 
à la vapeur. Thèse pour l'obtention du Diplôme d'Ingénieur Agronome Option : Nutrition et Sciences Alimentaires, Faculté des Sciences Agronomiques, Abomey-Calavi, 69p

Angladette A. 1966. Le riz. Techniques Agricoles et Productions Tropicales. Editions Maisonneuve et Larose: Paris.

AOAC. 1984. Approved methods of the American Association of Cereal Chemists ( $8^{\text {th }}$ edition). AOAC: USA.

CCRB. 2004. Initiatives de transformation et de commercialisation du riz au SudBénin. Comité de Concertation des Riziculteurs du Bénin. Interréseaux/CTA/PPAB. Bénin.

Ballogou YV. 2008. Evaluation des paramètres physicochimiques et microbiologiques du Chakpalo fementé à la levure commerciale et au ferment traditionnel et stabilisé par pasteurisation. Mémoire en vue de l'obtentention du Diplôme d'Etudes Approfondies, Université de Lomé, Togo, $63 \mathrm{p}$.

Dagan AA. 2006. Analyse des déterminants socio-organisationnels et institutionnels de l'adoption et de la diffusion du dispositif amélioré d'étuvage du riz dans la commune de Glazoué. Thèse pour l'obtention du diplôme d'ingénieur agronome, FSA/UAC Abomey-Calavi, $115 \mathrm{p}$.

Djakpo E. 2010. Test d'acceptabilité du ablo au riz local par le consommateur urbain dans la ville de Cotonou. Mémoire de Licence Professionnelle en Biotechnologie des Industries Alimentaires, UATM/GASA-FORMATION, Cotonou, $39 \mathrm{p}$.

Dossou J, Ahokpe F, Odjo DPS. 2008. Valorisation des ressources alimentaires locaux : Evaluation des procédés traditionnels de production du ablo, un pain humide cuit à la vapeur au Bénin. Communication scientifique présentée aux $4^{\text {eme }}$ journées de la SOACHIM. 1117 Août 2008, Université de Lomé, Togo, $12 \mathrm{p}$.

Dossou J, Ballogou YV. 2007. Analyse économique de la production du chakpalo au Bénin. Communication à l'atelier scientifique de l'INRAB, édition 2007 à Parakou, MAEP, Bénin, 15 p.

Dossou J, Egounlety M, Zohoun E, N'zamjo G. 2005. Analyse de l'incidence du coût de l'énergie de cuisson sur la marge brute du gari produit au sud du Bénin. $J$. Rech. Sci. Univ. Lomé, 7(1): 49-58.

FAO. 1997. Elaboration d'un plan de relance de la filière riz. Rapport définitif, vol 1. FAO/TCP/BEN/5613(A) Cotonou, Bénin.

FAO/OMS. 2005. Le secteur informel de la distribution de produits alimentaires vendus sur la voie publique: importance et enjeux. Conférence régionale FAO/OMS sur la sécurité sanitaire des aliments pour l'Afrique Harare, Zimbabwe, 3-6 octobre 2005 (Document préparé par le Zimbabwe). FAO/OMS Rome, $11 \mathrm{p}$.

Gariboldi F. 1986. L'étuvage du riz. Bulletin des services agricoles de la FAO $n^{\circ} 56$, $F A O$, Rome, Italie.

Gbenou P. 2004. Situation de la filière riz et des initiatives paysannes au Bénin. Conseil de Concertation des Riziculteurs du Bénin (CCRB) Cotonou Bénin.

Hounhouigan DJ. 1994. Fermentation of maize (Zea mays L.) meal for mawè production in Benin: Physical, chemical and microbiological aspects. PhD Thesis, Agricultural University, Wageningen, The Netherlands, 99p.

Hounhouigan DJ. 1997. La transformation des céréales : maïs, sorgho et mil. Manuel de référence pour TSTAC. Cotonou, Bénin.

Hounhouigan DJ. 2003. La valorisation des céréales locales pour les marchés urbains en Afrique de l'Ouest: atouts, contraintes et perspectives 2ème Atelier 
international / 2nd International Workshop Voies alimentaires d'amélioration des situations nutritionnelles Food-based approaches for a healthy nutrition Ouagadougou, 23$28 / 11 / 2003$

Hounhouigan D J. 2006. Qualité de quelques types de riz vendus au Bénin. Rapport d'expertise. Comité de Concertation des Riziculteurs du Bénin (CCRB). Bénin.

Houssou P. 2002. Développement de l'étuvage du riz au Bénin. In Savanes africaines. Des espaces en mutation, des acteurs face à de nouveaux défis, Jamin JY, Seiny Boukar L, Floret C. (eds). Actes du colloque, mai 2002, Garoua, Cameroun. Prasac, N'Djamena, Tchad Cirad, Montpellier, France. 9 p.

Houssou P. 2005. Valorisation des céréales au Bénin. Rapport de recherche. Institut National des Recherches Agricoles du Bénin (INRAB-PTAA), Bénin, 4 p.

INSAE. 2000 : Statistiques de consommation du riz au Bénin. Annuaire statistique; Ministère de l'économie et des Finances, Cotonou, Bénin.

Lees R. 1968. Laboratory Handbook of Methods of Food Analysis. Leonard hills : London.

MAEP. 2005. Politiques et stratégies mises en place par le MAEP pour promouvoir la production du riz et la sécurité alimentaire en République du Bénin. Présentation du MAEP à l'atelier sur les « Politiques et stratégies pour promouvoir la production du riz et la sécurité alimentaire en Afrique subsaharienne. Cotonou 7 au 9 Novembre 2005. Michodjehoun-Mestres L, Hounhouigan DJ, Dossou J, Mestres C. 2005. Physical, chemical and microbiological changes during natural fermentation of «gowé», a fermented beverage from sprouted or non sprouted sorghum (Sorghum bicolor). West-Africa. Afr. J. Biotech., 4: 487-496.

Mugula JK, Nnko SAM, Narvhus JA, Sorhaug T. 2002. Microbiological and fermentation characteristics of togya, a Tanzanian fermented food. International Journal of food microbiology, 80: 187199

Nago CM. 1989. Technologies traditionnelles et alimentation au Bénin: aspects techniques, biochimiques et nutritionnels. 1-Identification et caractérisation des principales filières et technologies du secteur traditionnel de transformation alimentaire. Document FSA/UNB, Abomey-Calavi, Bénin, 97p.

Nago MC, Hounhouigan J D.1994. Transformation traditionnelle du maïs au Bénin : aspects technologiques et socioéconomiques. In CIRAD Production et valorisation du maïs à l'échelon villageois en Afrique de l'Ouest. Actes $\mathrm{du}$ séminaire «Maïs prospère» 25-28 janvier 1994, Cotonou, Bénin.

Nago CM. 1997. La transformation alimentaire traditionnelle du maïs au Bénin : détermination des caractéristiques physico-chimiques des variétés en usage; relations avec l'obtention et la qualité des principaux produits dérivés. Thèse de doctorat d'Etat Es-Science, Université Paris VIIDenis Diderot, 571p.

Nago CM , Hounhouigan DJ. 1998. La Transformation Alimentaire des Céréales au Bénin. Les Publications du CERNA : 150p.

Niche PF, Nout MJR, Rombouts FM. 1994. The effect of cowpea supplementation on the quality of kenky, a traditional ghanaian fermented food. J. Cereal Sci., 19: 191-197.

Nout MJR, Rombouts FM , Havelaar A. 1989. Effect of accelerated natural lactic fermentation of infant good ingredients 
on some pathogenic microorganism. Int. J. Food Microbiol., 8: 351-361.

Nout MJR. 1991. Ecology of accelerated natural lactic fermentation of sorghum based infant food formulas. Int. J. Food Microbiol., 12: 217-224

Nout R, Hounhouigan J, Van Boekel T. 2003. Les Aliments : Transformation, Conservation et Qualité. Backhuys Publishers CTA: Wageningen.

Odunfa SA, Adeyele S.1985. Microbial changes during traditional production of ogi-baba, a western Africa fermented sorghum gruel. J. Cereals Sci., 3: 173180.

Odjo DPS. 2008. Aptitudes de quelques variétés locales de riz à la production artisanale du ablo. Thèse d'Ingénieur Agronome, FSA/UAC, Bénin, 93 p.
ONASA. 2005a : Evaluation de la production vivrière en 2004 et les perspectives alimentaires pour 2005 au Bénin, Rapport d'étude, Vol 1.MAEP, Cotonou, $45 \mathrm{p}$.

ONASA. 2005b : Evaluation de la production vivrière en 2004 et les perspectives alimentaires pour 2005 au Bénin, Rapport d'étude, Vol 2. MAEP, Cotonou, 149 p.

Verlinden E, Soulé BG. 2003. Etude de la Filière Riz au Bénin: Diagnostic Plan d'Action. PADSE /SOFRECO. 102p. 